\title{
PERIODIC AND RECURSIVE CONTROL THEORETIC SMOOTHING SPLINES
}

\author{
MAJA KARASALO*, XIAOMING HU*, AND CLYDE F. MARTIN ${ }^{\dagger}$
}

\begin{abstract}
In this paper, a recursive control theoretic smoothing spline approach is proposed for reconstructing a closed contour. Periodic splines are generated by minimizing a cost function subject to constraints imposed by a linear control system. The optimal control problem is shown to be proper, and sufficient optimality conditions are derived for a special case of the problem using Hamilton-Jacobi-Bellman theory.

The filtering effect of the smoothing splines allows for usage of noisy sensor data. An important feature of the method is that several data sets for the same closed contour can be processed recursively so that the accuracy is improved stepwise as new data becomes available.
\end{abstract}

Keywords: Smoothing splines, optimal control, Hamilton-Jacobi-Bellman theory, periodic solutions.

1. Introduction. In this paper we focus on the problem of reconstructing closed contours from noisy and sparse samples.

Data smoothing is a classical problem in system and control history $[1,3]$. Smoothing splines were introduced in the 1960s. As opposed to interpolating splines, smoothing splines pass close to, rather than through interpolation points, providing a filtering or smoothing effect. A comprehensive overview is given in [4] and [5], where splines are studied in a statistical setting. In mathematical statistics, the aim of the smoothing spline is generally to fit a curve to a data set so that the error between the curve and the data has nice statistical properties, for instance that it is normally distributed.

Control theoretic smoothing splines were first introduced in [10], and are further studied in for instance [11, 12, 15]. In [16], the smoothing spline problem is solved using Hilbert space methods. [6] is, to the extent of the authors' knowledge, the first book to give a complete overview of the field. The aim of control theoretic smoothing splines is to find a tradeoff between faithfulness to a given data set and control gain. This is motivated by for instance trajectory tracking applications, where exact tracking of way points often calls for undesirable large accelerations.

A nice property of smoothing splines is robustness. [13] observes that smoothing splines are in some sense band limited so that small changes in one data point will mainly affect the spline in a neighborhood of that point.

\footnotetext{
*Optimization and Systems Theory, Royal Institute of Technology, Stockholm, Sweden. E-mail: $\{$ karasalo,hu\}@math.kth.se

$\dagger$ Mathematics and Statistics, Texas Tech University, Lubbock, Texas. E-mail: clyde.f.martin@ttu.edu
} 
The focus of this paper is control theoretic smoothing splines with a periodicity constraint. A recursive approach is developed, where the estimate of the underlying, closed curve is improved stepwise as new data sets are recovered.

We note that recursive approaches to constructing splines have been investigated in [23] and [29]. In the field of robotics, recursive cubic B-spline methods for path planning have been presented in [21] and [22]. However, little work has been done in this direction with control theoretic splines. A notable contribution is presented in [6]. While the recursive smoothing spline problem formulated in [6] includes previous curve estimates, the formulation in the present paper only includes them implicitly.

Control theoretic smoothing splines may be viewed as point-to-point LQ optimal control problems with dynamic constraints. Point-to-point LQ optimal control problems have been investigated in for instance [24] and [25], where [24] treats the optimal output-transition problem for linear systems while [25] considers LTI continuous-time systems with affine constraints in initial and terminal states.

[14] investigates a point-to-point LQ optimal control problem under the assumption of dynamic constraints with a stochastic uncertainty. This paper examines a similar LQ problem, with the important distinction that we optimize over all periodic solutions. Again, results for periodic LQ problems can be found in papers dating back to the $1970 \mathrm{~s},[8,9]$, but these do not cover the point-to-point problem.

In the field of mathematical statistics, early contributions on periodic smoothing splines include [26]. Until recently, little work had been done on periodic control theoretic smoothing splines. Notable contributions have however emerged during the past few years. [28] studies applications of control theoretic smoothing splines to mobile robotics, and poses a problem where the periodicity constraint depends on input data. In [27] periodic control theoretic smoothing spline problems are solved using Hilbert Space methods. In the current paper, optimality conditions for such splines are examined using Hamilton-Jacobi-Bellman theory for optimal control problems.

The paper is organized as follows. In Section 2, we state the contour estimation problem formally and propose a closed form and a recursive point-to-point LQ formulation for estimation of closed contours. In Section 3 we discuss optimality conditions for the periodic smoothing spline problem. Some simulation results are reported in Section 4 , and a concluding summary is provided in Section 5 .

2. Problem statement and motivation. Consider the problem of reconstructing continuous, smooth, closed curves in $\mathbb{R}^{2}$ from noisy and sparse measurement data. This problem arises for instance in mapping applications and trajectory tracking for mobile robots. In this section we pose two problems that aim to find the best estimate of an underlying, closed curve given noise contaminated samples from the curve. First, we introduce a closed form optimal control problem that yields an initial estimate of the underlying curve and then a modified, recursive problem. A formal 
problem statement follows.

Given a data set $D=\left\{\left(t_{i}, z_{i}\right): i=1, \ldots, N\right\}$, where $z_{i}=z\left(t_{i}\right), t_{i} \in[0, T]$ and $z(T)=z(0)$. If $z_{i}=y\left(t_{i}\right)+\xi_{i}$ are noise contaminated samples from a closed continuous curve, where $\xi_{i}$ is a symmetric, zero-mean iid noise with bounded variance, how to find the function $y(t)$ that best represents the underlying curve, with respect to smoothness and closeness to measurement data?

If $y(t)$ is viewed as the output of a dynamic system with input $u(t)$, an optimal control approach can be used.

2.1. Closed Form. The following closed form $L_{2}$ smoothing problem yields an estimate of $y(t)$ while minimizing the control effort $u(t)$.

Problem 2.1.

(1) $\underset{u \in L_{2}[0, T]}{\operatorname{minimize}} J(u, x)=\frac{1}{2} \int_{0}^{T} u(t)^{\prime} Q^{-1} u(t) d t+$

$$
\frac{1}{2} \sum_{i=1}^{N}\left(t_{i}-t_{i-1}\right)\left(z_{i}-y\left(t_{i}\right)\right)^{\prime} R^{-1}\left(z_{i}-y\left(t_{i}\right)\right)
$$

$$
\begin{aligned}
\text { subject to } \quad \dot{x} & =A x+B u \\
y & =C x \\
x(0) & =x(T),
\end{aligned}
$$

where $t_{0}=t_{N}-T$, and $t_{i}>t_{i-1}$ for $i=1, \ldots, N$. (.)' denotes the transpose of (.). $A \in \mathbb{R}^{n \times n}, B \in \mathbb{R}^{n}$ and $C^{\prime} \in \mathbb{R}^{n}$, where the pair $(A, B)$ is controllable and $(A, C)$ is observable. $Q$ and $R$ are positive definite matrices of suitable dimension. The resulting smoothing spline is given by $y(t)=C x(t)$.

Let us have a closer look at the cost function (1). The integral imposes a penalty on large magnitude of the input $u(t)$. The sum punishes large deviations of the curve $y(t)$ from the data $\left(t_{i}, z_{i}\right)$. In other words, the solution of Problem 2.1 is in some sense the optimal compromise between low control gain and faithfulness to the data set. The magnitude of the components of $R^{-1}$ and $Q^{-1}$ determine the tradeoff between these two conflicting objectives.

The system (2) - (3) is referred to as the spline generator of Problem 2.1. The relative degree of the spline generator determines on which derivative to impose the smoothing penalty.

EXAMPLE 2.1. For many practical problems, a natural choice is to impose the smoothing penalty on the second derivative of the spline. This corresponds to a system 
with relative degree 2. The simplest representation of such a system is

$$
\begin{array}{cc}
A=\left[\begin{array}{ll}
0 & 1 \\
0 & 0
\end{array}\right], \quad B=\left[\begin{array}{l}
0 \\
1
\end{array}\right], \quad C=\left[\begin{array}{ll}
1 & 0
\end{array}\right], \\
Q=1, & R=1 / \varepsilon^{2}, \quad \varepsilon>0 \quad T=2 \pi .
\end{array}
$$

In the next section it is shown how a modification of Problem 2.1 allows for a recursive approach to the contour estimation problem.

2.2. Recursive Form. As the data is noise contaminated, the resulting spline from one data set $D$ may give a poor estimate of the underlying curve. If new data becomes available over time, improvements of the estimate can be made by solving a recursive form of Problem 2.1. Here, the optimal control $u^{k-1}(t)$ from the previous iteration is used in iteration $k$ together with new data $\left(t_{i}^{k}, z_{i}^{k}\right)$.

PROBLEM 2.2.

(6) $\underset{u^{k} \in L_{2}[0, T]}{\operatorname{minimize}} J^{k}\left(u^{k}, x^{k}\right)=\frac{1}{2} \int_{0}^{T}\left(u^{k}(t)-u^{k-1}(t)\right)^{\prime} Q^{-1}\left(u^{k}(t)-u^{k-1}(t)\right) d t+$

$$
\frac{1}{2} \sum_{i=1}^{N}\left(t_{i}^{k}-t_{i-1}^{k}\right)\left(z_{i}^{k}-y^{k}\left(t_{i}^{k}\right)\right)^{\prime} R^{-1}\left(z_{i}^{k}-y^{k}\left(t_{i}^{k}\right)\right)
$$

$$
\begin{aligned}
\text { subject to } \quad \dot{x}^{k} & =A x^{k}+B u^{k} \\
y^{k} & =C x^{k} \\
x^{k}(0) & =x^{k}(T) .
\end{aligned}
$$

Introduce the notation

$$
\begin{aligned}
\tilde{z}_{i}^{k} & =z_{i}^{k}-y^{k-1}\left(t_{i}^{k}\right) \\
\tilde{x}^{k}(t) & =x^{k}(t)-x^{k-1}(t) \\
\tilde{y}^{k}(t) & =y^{k}(t)-y^{k-1}(t) \\
\tilde{u}^{k}(t) & =u^{k}(t)-u^{k-1}(t) .
\end{aligned}
$$

Substituting for the variables (10) in (6) - (9), we obtain

$$
\begin{aligned}
\underset{\tilde{u}^{k} \in L_{2}[0, T]}{\operatorname{minimize}} J^{k}\left(\tilde{u}^{k}, \tilde{x}^{k}\right)=\frac{1}{2} \int_{0}^{T} \tilde{u}^{k}(t)^{\prime} Q^{-1} \tilde{u}^{k}(t) d t+ \\
\frac{1}{2} \sum_{i=1}^{N}\left(t_{i}^{k}-t_{i-1}^{k}\right)\left(\tilde{z}_{i}^{k}-\tilde{y}^{k}\left(t_{i}\right)\right)^{\prime} R^{-1}\left(\tilde{z}_{i}^{k}-\tilde{y}^{k}\left(t_{i}\right)\right) \\
\dot{\tilde{x}}^{k}=A \tilde{x}^{k}+B \tilde{u}^{k} \\
\tilde{y}^{k}=C \tilde{x}^{k} \\
\tilde{x}^{k}(0)=\tilde{x}^{k}(T),
\end{aligned}
$$


which are identical to (1) - (4). Therefore, solution methods and optimality conditions for Problem 2.1 and Problem 2.2 are identical. At iteration $k$, the spline solution $\tilde{y}^{k}$ of Problem 2.2 is an adjustment of the curve $y^{k-1}$ based on the new data $\left(t_{i}^{k}, z_{i}^{k}\right)$. The curve estimate at iteration $k$ can now be written

$$
y^{k}(t)=y^{1}(t)+\sum_{j=2}^{k-1} \tilde{y}^{j}(t),
$$

where $y^{1}(t)$ is the spline solution to Problem 2.1 for the first batch of data. In the next section, optimality conditions for this smoothing problem is discussed.

REMARK 2.1. It is intuitively easy to see that using the closed form (Problem 2.1) and increasing the number $N$ of data points, the spline output should approach the underlying curve if the noise is symmetric and the variance is bounded. If such a data set is available, this may be an option. In many applications, however, new data may arrive at different points in time, calling for an update of the estimate. This is for instance the case when data is collected by teams of cooperating autonomous vehicles. As $k$ increases, the error of the spline estimate with this recursive formulation decreases only slightly slower than when increasing $N$ for the closed form. In the extreme, a further motivation for the recursive formulation is that as $N \rightarrow \infty$, Problem 2.1 may experience numerical instability in implementation.

3. Properness and Optimality. Problem 2.1 is a continuous time problem with discrete data and periodic boundary condition. Such problems, without the periodicity constraint, have been widely studied in the literature, see for example the books by Bryson and Ho [1], Leondes [2] and Jazwinski [3]. However, as far as we know, it is difficult to find results concerning the periodic case. In this section, we investigate conditions for solving this problem. We begin by studying the proper periodicity conditions.

3.1. Proper periodicity conditions. In this section, we adopt the notations used in [7]. Let $\bar{J}^{*}=J\left(\bar{u}^{*}, \bar{x}^{*}\right)$ in Problem 2.1, where $\bar{u}^{*}$ is any constant function.

DEFINITION 3.1. The optimal control problem is proper if there exists an admissible control $\bar{u}(\cdot)$ such that

$$
J(\bar{u}(\cdot), \bar{x}(\cdot))<\bar{J}^{*}
$$

In this context, proper periodicity conditions refers to conditions determining whether an optimal, periodic control problem is proper or not. In this paper we will only discuss properness for systems of the form (5), since, as previously mentioned, for many practical problems this model represents the desired constraints on the spline. The following proposition establishes that Problem 2.1 is proper for all but a special case of data input.

Proposition 3.1. For distinct sampling times $\left[t_{1}, \ldots, t_{N}\right]$, Problem 2.1, with the numerical values of (5), is proper if and only if $\exists i, j \in[1, N]$ such that $z_{i} \neq z_{j}$. 
Proof. The proof is constructed by showing that a particular, time varying, periodic control $\bar{u}(t)=\alpha \hat{u}(t)$ satisfies (16). For details, see the Appendix.

Next, we discuss optimality conditions for Problem 2.1.

3.2. Optimality Conditions and Hamilton-Jacobi-Bellman Theory. In the following, sufficient optimality conditions for Problem 2.1 are examined using a Hamilton-Jacobi-Bellman approach. A set of differential equations is derived, leading to an expression for the optimal control $u$. It should be noted that for the particular choice of system matrices (5), straightforward and well known approaches for regular smoothing splines are applicable [5]. The purpose of this section is to analyze smoothing splines from a control perspective and suggest solutions for more complex spline generators.

First, a brief review of the Hamilton-Jacobi-Bellman theory is given in Section 3.2.1. Then, in Section 3.2.2, sufficient optimality conditions are given for the special case of Problem 2.1 where the input is a continuous curve. Finally, the discrete data case is discussed in Section 3.2.3.

3.2.1. Optimality Conditions for a General Periodic Control Problem. In this section we will study a general optimal control problem in order to introduce notation and state a proposition regarding optimality conditions in a general case. Subsequently, we will study the special case of LQ optimal control problems more closely.

Consider the problem

PROBLEM 3.1.

$$
\begin{gathered}
\underset{u \in L_{2}[0, T]}{\operatorname{minimize}} J(u, x)=\int_{0}^{T} L(x(t), u(t)) d t \\
\text { subject to } \quad \dot{x}=f(x, u) \\
x(0)=x(T) .
\end{gathered}
$$

Here $x \in \mathbb{R}^{n}$. We assume that $L(x(t), u(t))$ and $f(x, u)$ are $T$-periodic functions. Some definitions of useful concepts follow.

Definition 3.2. The Hamiltonian of Problem 3.1 is

$$
H(x, u, \lambda) \triangleq L(x, u)+\lambda^{\prime} f(x, u) .
$$

Definition 3.3. The $\boldsymbol{H}$-minimal control $u^{*}$ is defined as

$$
u^{*}(x, \lambda) \triangleq \underset{u}{\arg \min } H(x, u, \lambda)
$$


Definition 3.4. Let $V:[0, T] \times \mathbb{R}^{n} \mapsto \mathbb{R}$. Then the Hamilton-Jacobi-Bellman equation is

$$
\frac{\partial V(t, x)}{\partial t}=-H\left(x, u^{*}\left(x, \frac{\partial V(t, x)}{\partial x}\right), \frac{\partial V(t, x)}{\partial x}\right) .
$$

The following proposition is proved in [8]:

Proposition 3.2. Suppose that

1. The control $u_{T}$ generates a periodic solution $x_{T}$ to Problem 3.1.

2. There exists a continuously differentiable solution $V(t, x)$ of $(22)$ such that

$$
V(0, x)-V(T, x)=G(T)
$$

where $G(t)$ is a real function.

Then, $u_{T}$ is optimal to Problem 3.1 if

$$
u_{T}=u^{*}\left(x_{T}, \frac{\partial V\left(t, x_{T}\right)}{\partial x}\right) .
$$

Next, optimality conditions are first stated for a periodic smoothing problem with continuous data and a general expression for the optimal control is given. Finally, the optimal control for Problem 2.1 in the limit $N \rightarrow \infty$ is derived.

3.2.2. Optimality Conditions: Continuous time, continuous data. Consider the following problem:

Problem 3.2.

$$
\begin{aligned}
\underset{u \in L_{2}[0, T]}{\operatorname{minimize}} J(u, x)=\frac{1}{2} \int_{0}^{T}\left[u(t)^{\prime} Q^{-1} u(t)+(z(t)-y(t))^{\prime} R^{-1}(z(t)-y(t))\right] d t \\
\text { subject to } \begin{aligned}
\dot{x} & =A x+B u \\
y & =C x \\
x(0) & =x(T) .
\end{aligned}
\end{aligned}
$$

Here $z(t)$ is a continuous periodic function such that $z(0)=z(T)$. This may be viewed as a smoothing problem with continuous data $z(t)$ or as a problem of tracking a curve given by $z(t)$. Optimality conditions for problems of this type were derived in [8]. A review of the results follows. For brevity, throughout this section we use the notation $V_{t}$ and $V_{x}$ to denote the partial derivatives of $V$. The Hamiltonian corresponding to $(25)$ is, since $y=C x$,

$$
H(x, u, \lambda)=\frac{1}{2} u^{\prime} Q^{-1} u+\frac{1}{2} x^{\prime} C^{\prime} R^{-1} C x-x^{\prime} C^{\prime} R^{-1} z+\frac{1}{2} z^{\prime} R^{-1} z+\lambda^{\prime}(A x+B u) .
$$


The $H-$ minimal control $u^{*}$ is derived as

$$
\frac{\partial H(x, u, \lambda)}{\partial u}=Q^{-1} u+B^{\prime} \lambda \quad \Rightarrow \quad u^{*}=-Q B^{\prime} \lambda,
$$

so $u^{*}\left(x_{T}, V_{x}\right)=-Q B^{\prime} V_{x}$. Then the Hamilton-Jacobi-Bellman equation is

$$
V_{t}=\frac{1}{2} V_{x}^{\prime} B Q B^{\prime} V_{x}-\frac{1}{2} x^{\prime} C^{\prime} R^{-1} C x+x^{\prime} C^{\prime} R^{-1} z-\frac{1}{2} z^{\prime} R^{-1} z-V_{x}^{\prime} A x .
$$

In [8] the following form for $V(t, x)$ is proposed for Problem 3.2:

$$
V(t, x)=\frac{1}{2} x^{\prime} P x+x^{\prime} \phi+s .
$$

Here, $\phi, P$ and $s$ should be chosen so that (31) is satisfied, and $V(0, x)-V(T, x)=$ $G(T)$ for some real function $G(t)$. Furthermore, $P \in \mathbb{R}^{n \times n}$ is a symmetric, positive semidefinite matrix. The derivatives $V_{t}$ and $V_{x}$ are

$$
\begin{aligned}
V_{t} & =\frac{1}{2} \dot{x}^{\prime} P x+\frac{1}{2} x^{\prime} P \dot{x}+\frac{1}{2} x^{\prime} \dot{P} x+\dot{x}^{\prime} \phi+x^{\prime} \dot{\phi}+\dot{s} \\
V_{x} & =P x+\phi .
\end{aligned}
$$

Then, since

$$
u_{T}=u^{*}\left(x, V_{x}\right)=-Q B^{\prime}(P x+\phi)
$$

we get

$$
\dot{x}_{T}=A x_{T}+B u_{T}=A x_{T}-B Q B^{\prime}\left(P x_{T}+\phi\right)=\left(A-B Q B^{\prime} P\right) x_{T}-B Q B^{\prime} \phi .
$$

Somewhat tedious calculations yield, after plugging in (33), (34) and (35) into (31), that $P, \phi$ and $s$ must satisfy

$$
\begin{aligned}
\dot{P} & =-A^{\prime} P-P A+P B Q B^{\prime} P-C^{\prime} R^{-1} C \\
\dot{\phi} & =-\left(A-B Q B^{\prime} P\right)^{\prime} \phi+C^{\prime} R^{-1} z \\
\dot{s} & =\frac{1}{2} \phi^{\prime} B Q B^{\prime} \phi-\frac{1}{2} z^{\prime} R^{-1} z \\
P(T) & =P(0) \\
\phi(T) & =\phi(0) \\
s(T) & =s(0)-G(T) .
\end{aligned}
$$

A brief discussion of the existence of solutions to (37) - (42) is provided next. The following proposition is proved in [31].

Proposition 3.3. Under the assumption that $Q>0$ and $R>0$, the Riccati equation (37) admits a unique, T-periodic, stabilizing solution $P(t)=P(t)^{\prime} \geq 0$ if and only if the pair $(A, B)$ is stabilizable and the pair $(A, C)$ is detectable. 
Now, consider the system

$$
\left(\begin{array}{c}
\dot{x} \\
\dot{\phi}
\end{array}\right)=\left(\begin{array}{cc}
A-B Q B^{\prime} P(t) & -B Q B^{\prime} \\
0 & -\left(A-B Q B^{\prime} P(t)\right)^{\prime}
\end{array}\right)\left(\begin{array}{l}
x \\
\phi
\end{array}\right)+\left(\begin{array}{c}
0 \\
C^{\prime} R^{-1} z(t)
\end{array}\right) .
$$

Denote by $\mathbf{X}(t)$ the matrix in (43). The following proposition is proved in [32].

Proposition 3.4. Under the assumption that the components of $\mathbf{X}(t)$ are Lebesque integrable, and $X(0)=X(T), z(0)=z(T)$, the system (43) has a unique periodic solution $(x(t) \phi(t))^{\prime}, x(0)=x(T), \phi(0)=\phi(T)$ if and only if

$$
\operatorname{det}\left(I-e^{\mathbf{X}(T)}\right) \neq 0
$$

where $I$ is the identity matrix of suitable dimension.

3.2.3. Optimality Conditions: Continuous time, discrete data. We restate Problem 2.1 for the reader's convenience:

PRoblem 3.3.

$$
\begin{aligned}
\underset{u \in L_{2}[0, T]}{\operatorname{minimize}} J(u, x)=\frac{1}{2} \int_{0}^{T} u(t)^{\prime} Q^{-1} u(t) d t+ \\
\frac{1}{2} \sum_{i=1}^{N}\left(t_{i}-t_{i-1}\right)\left(z\left(t_{i}\right)-y\left(t_{i}\right)\right)^{\prime} R^{-1}\left(z\left(t_{i}\right)-y\left(t_{i}\right)\right) \\
\text { subject to } \quad \dot{x}=A x+B u \\
y=C x \\
x(0)=x(T) .
\end{aligned}
$$

Note that here, we represent the data $z_{i}$ as samples of a function $z(t)$ at times $t_{i}$. Due to the periodicity constraint, it is not trivial to find optimality conditions for this problem. A similar problem is studied in [14]. There, the cost function is of the form

$$
\sum_{i=0}^{N-1}\left(w_{i+1}\left\|z\left(t_{i+1}\right)-C x\left(t_{i+1}\right)\right\|^{2}+\int_{t_{i}}^{t_{i+1}} \sigma_{i+1}(t, x, u) d t\right)
$$

where $\sigma_{i}(t, x, u)$ contains, in addition to the $u$-quadratic term, cross terms for $x$ and $u$ and linear terms in $x$ and $u$. [14] assumes dynamic constraints of the form (46) but includes a multiplicative stochastic uncertainty. Further, the initial value $x(0)$ is fix.

(45) may be viewed as a special case of (49), where some terms are removed. Removing the stochastic terms from the dynamic constraints, the control problem in [14] is the same as Problem 3.3, except for the boundary constraints. In [14], using 
functions $V_{i}(t, x)$ of the form (32), the following relations are obtained for each interval $\left(t_{i}, t_{i+1}\right]$ (where we have substituted for the notation in the current paper):

$$
\begin{aligned}
\dot{P}_{i} & =-A^{\prime} P_{i}-P_{i} A+P_{i} B Q B^{\prime} P_{i} \\
P_{i}\left(t_{i+1}\right) & =P_{i+1}\left(t_{i+1}\right)+\left(t_{i+1}-t_{i}\right) C^{\prime} R^{-1} C \\
\dot{\phi}_{i} & =-\left(A-B Q B^{\prime} P_{i}\right)^{\prime} \phi_{i} \\
\phi_{i}\left(t_{i+1}\right) & =\phi_{i+1}\left(t_{i+1}\right)-\left(t_{i+1}-t_{i}\right) C^{\prime} R^{-1} z\left(t_{i+1}\right) \\
\dot{s}_{i} & =\frac{1}{2} \phi_{i}^{\prime} B Q B^{\prime} \phi_{i} \\
s_{i}\left(t_{i+1}\right) & =s_{i+1}\left(t_{i+1}\right)+\frac{1}{2}\left(t_{i+1}-t_{i}\right) z\left(t_{i+1}\right)^{\prime} R^{-1} z\left(t_{i+1}\right) .
\end{aligned}
$$

For $(A, B)$ controllable, $(A, C)$ observable and $Q>0, R>0$, the Riccati equation (50) has an absolutely continuous unique positive semidefinite solution for each $i$ [30]. The differential equations (52) and (54) are linear with bounded piecewise continuous coefficients, which ensure existence of unique absolutely continuous solutions. On the interval $\left(t_{i}, t_{i+1}\right]$ the resulting optimal control is

$$
u_{i}(t)=-Q B^{\prime}\left(P_{i}(t) x(t)+\phi_{i}(t)\right) .
$$

The authors believe that Proposition 3.2 can be generalized to allow for a piecewise continuous function of the form

$$
V^{N}(t, x)=V_{i}(t, x) \quad t \in\left(t_{i}, t_{i+1}\right], \quad i=0, \ldots, N-1
$$

such that

$$
V^{N}(x, 0)-V^{N}(x, T)=G(T) .
$$

This would imply that the piecewise continuous control

$$
u_{T}^{N}(t)=u_{i}(t) \quad t \in\left(t_{i}, t_{i+1}\right], \quad i=0, \ldots, N-1
$$

is optimal to Problem 3.3 if there exist solutions to (50) - (55) such that

$$
\begin{aligned}
P^{N}(T) & =P^{N}(0) \\
\phi^{N}(T) & =\phi^{N}(0) \\
s^{N}(T) & =s^{N}(0)-G(T),
\end{aligned}
$$

with $P^{N}(t), \phi^{N}(t), s^{N}(t)$ defined analogously with $V^{N}(t, x)$ in (57). Although simulation results support this claim, a proof is yet to be constructed. A weaker result is stated in the following proposition.

Proposition 3.5. Let $N \rightarrow \infty$ in such a manner that $t_{i+1}-t_{i} \rightarrow 0 \forall i$. Then, under the assumption that (60) - (62) hold, $u_{T}^{N}$ defined by (59) converges to $u_{T}$ defined by (35). 
Proof. A proof is given in the appendix.

Remark 3.1. With a clever choice of discretization of Problem 3.3, $u_{T}^{N}$ can be computed as the solution of an unconstrained, quadratic programming problem, avoiding the difficulty of finding solutions to (50) - (55). This is discussed in further detail in [17], [18], [19] and [20].

4. Simulations. In this section, we show an example of curve estimation with Problem 2.1 and Problem 2.2. The numerical values (5) were used throughout the simulations. We let $y_{\text {true }}(t)$ denote the underlying curve while $\left(y^{k}(t), u_{T}^{N, k}(t)\right)$ denotes the optimal smoothing solution at iteration $k$. For $k=1$ the closed form (Problem 2.1) was used, and for $k=2,3, \ldots$ the recursive form (Problem 2.2), yielding the control $\tilde{u}_{T}^{N, k}(t)$, defined by (10). Throughout the simulations, a noise $\xi_{i} \in \mathbf{N}(0,0.05)$ was added to the samples $z_{i}^{k}$ of $y_{\text {true }}\left(t_{i}^{k}\right)$ to simulate measurement noise. An example is shown in Figure 1 for $k=1,5,10$. As expected, the resulting control $u_{T}^{N}$ defined by (59) is periodic with breaks at the interpolation points $t_{i}$. It is also worth noting that as $k$ grows, the magnitude of $\tilde{u}^{N, k}(t)$ decreases as a consequence of the error convergence.

To further evaluate the recursive problem, a study of error convergence was performed for Problem 2.2. For reference, Problem 2.1 was solved for an increasing number of data points $N$. Denote by $y^{k}(t)$ and $R^{k}$ the output curve and error of Problem 2.2 at iteration $k$ and let $y^{N}$ and $R^{N}$ denote the output curve and error of Problem 2.1 for $N$ data points. The errors were computed as

$$
R^{(\cdot)}=\int_{0}^{T}\left(y^{(\cdot)}(t)-y_{\text {true }}(t)\right)^{2} d t
$$

Results are shown in Figure 2. Mean values of $R^{k}$ and $R^{N}$ are plotted for 25 test cases. For $k=1, \ldots, 100$ Problem 2.2 was solved with $N(k)=15$ data points at each iteration, while Problem 2.1 was solved with $N(k)=15 k$ data points. Figure 2 shows that the performance of the recursive problem is almost as good as that of the closed form, without the drawbacks discussed in Remark 2.1.

5. Conclusions. In this paper, we introduced a recursive smoothing spline approach to estimation of closed curves in $\mathbb{R}^{2}$. We derived periodic smoothing splines recursively from noisy data by solving an optimal control problem for a linear system. It was shown that a simple, linear transform makes the recursive formulation identical to the closed form and that the problem is proper generically. Optimality conditions were examined using Hamilton-Jacobi-Bellman theory and simulations demonstrate satisfying error convergence for the recursive method. 

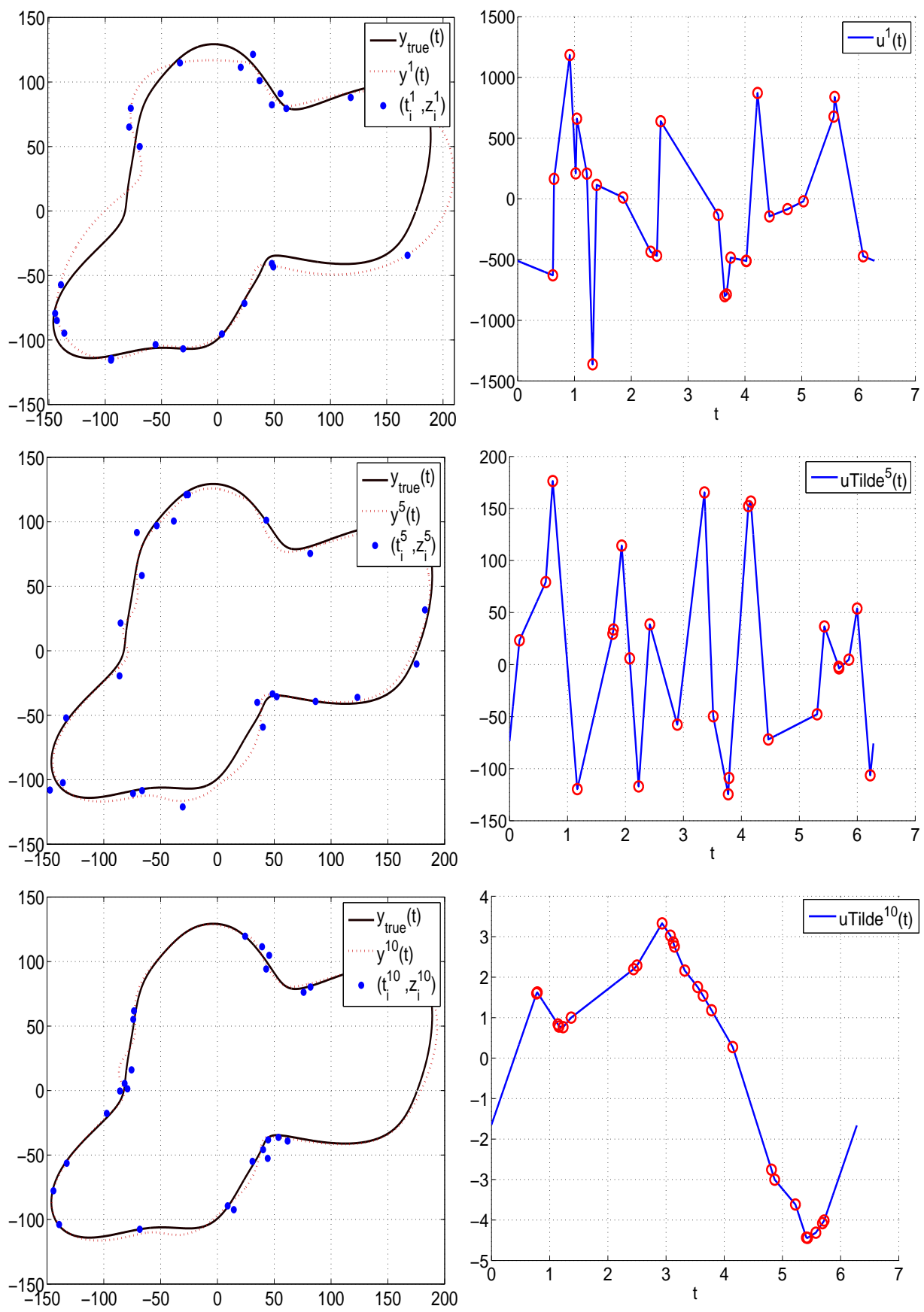

FIG. 1. An example. Left: Estimates $y^{k}(t)$ (dashed) are shown at iterations $k=1,5,10$ and compared with the underlying curve $y_{\text {true }}$ and the sampled input data $\left(t_{i}^{k}, z_{i}^{k}\right)$ (dots). Right: The corresponding controls $u_{T}^{N, 1}, \tilde{u}_{T}^{N, 5}$ and $\tilde{u}_{T}^{N, 10}$. The interpolation points at $t=t_{i}^{k}$ are marked with circles. 


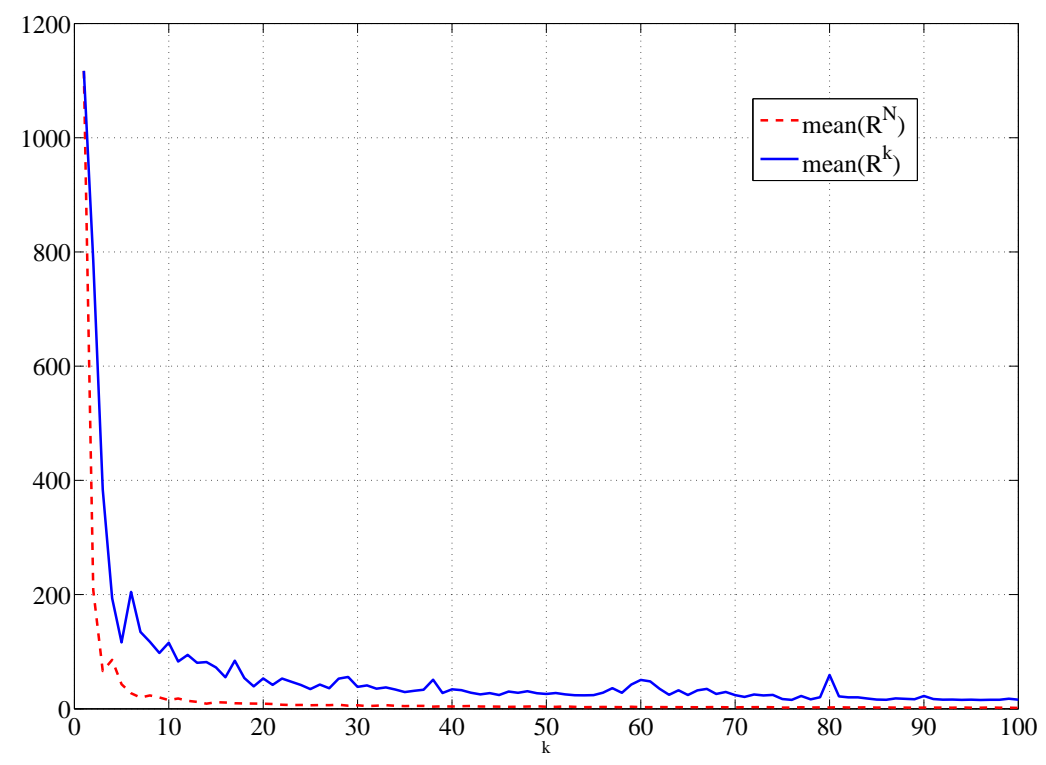

FIG. 2. Convergence results for Problem 2.2 for increasing $k$ (solid) compared to Problem 2.1 for increasing $N$ (dashed).

\section{REFERENCES}

[1] A.E. Bryson and Yu-Chi Ho, Applied Optimal Control, Optimization estimation and control, Halsted Press, 1975.

[2] C.T. Leondes, Advances in Control Systems, vol. 16, Academic Press, 1965.

[3] A. Jazwinski, Stochastic Processes and Filtering Theory, Academic Press, 1970.

[4] G. WAhBA, Spline Models for Observational Data, CBMS-NSF Series, SIAM, 1990.

[5] R.L. Eubank, Nonparametric Regression and Spline Smoothing, 2nd ed., CRC Press, 1999.

[6] M. Egerstedt and C.F. Martin, Control Theoretic Splines: Optimal control, statistics and path planning, Princeton Series in Applied Mathematics, to appear 2010.

[7] S. Bittanti, G. Fronza, and G. Guardabssi, Periodic Control: A Frequency Domain Approach, IEEE Trans. on Automatic Control, 18:1(1973).

[8] C. Maffezzoni, Hamilton-Jacobi Theory for Periodic Control Problems, Journal of Optimization Theory and Applications, 14:1(1974).

[9] S. Bittanti, A. Locatelli, and C. Maffezzoni, Second-Variation Methods in Periodic Optimization, Journal of Optimization Theory and Applications, 14:1(1974).

[10] C. F. Martin S. Sun, And M. Egerstedt, Control theoretic smoothing splines, IEEE Trans. on Automatic Control, 45(2000), pp. 2271-2279.

[11] M. Egerstedt, C. F. Martin, And S. Sun, Optimal control, statistics and path planning, Mathematical and Computer Modelling, 33(2001), pp. 237-253.

[12] M. Egerstedt And C.F. MARTin, Statistical estimates for generalized splines, ESAIM:Control, Optimisation and Calculus of Variations, 9(2003), pp. 553-562.

[13] Y. Zhou, W. Dayawansa, And C.F. Martin, Control theoretic smoothing splines are approximate linear filters, Communications in Information and Systems, 4(2004), pp. 253-272.

[14] U. Jönsson, C. F. Martin, And Y. Zhou, Trajectory Planning for Systems with a Multiplica- 
tive Stochastic Uncertainty, International Journal of Control, 77:8(2004).

[15] H. Kano, H. Fujioka, M. Egerstedt, and C.F Martin, Optimal smoothing spline curves and contour synthesis, Proc. of the 16th Congress of the International Federation of Automatic Control (IFAC), 2005.

[16] Y. Zhou, M. Egerstedt, And C. Martin, Hilbert Space Methods for Control Theoretic Splines: A Unified Treatment, Communications in Information and Systems, 6:1(2006), pp. $55-82$.

[17] G. Piccolo, M. Karasalo, D. Kragic, and X. Hu, Contour Reconstruction using Recursive Smoothing Splines - Experimental Validation, Proc. of the IEEE/RSJ International Conference on Intelligent Robots and Systems (IROS), 2007.

[18] M. Karasalo, X. Hu, And C.F. Martin, Localization and Mapping using Recursive Smoothing Splines, Proc. of the Eurpoean Control Conference (ECC), 2007.

[19] M. Karasalo, G. Piccolo, D. Kragic, and X. Hu, Contour reconstruction using recursive smoothing splines - Algorithms and experimental validation, Robotics and Autonomous Systems, 57(2009), pp. 617-628.

[20] M. Karasalo, X. Hu, And C.F. Martin, Contour Reconstruction and Matching using Recursive Smoothing Splines, Modeling, Estimation and Control, Springer, pp. 193-206, 2007.

[21] R. Frezza, S. Soatto, And G. Picci, Visual Path Planning by Recursive Spline Updating, Proc. of the 36th Conference of Decision and Control (CDC), pp. 367-393, 1997.

[22] R. Frezza and G. Picci, On Line Path Following by Recursive Spline Updating, Proc. of the 34th Conference of Decision and Control (CDC), pp. 367-393, 1995.

[23] S. Isotani, A. de Albuquerque, M. Muratore, and N. Brasil Filho, A recursive splinebased algorithm for sensor calibration design, Industrial Electronics, Control and Instrumentation, 3(1994), pp. 1952-1954.

[24] H. Perez And S. Devasia, Optimal output-transitions for linear systems, Automatica, 39:2(2003), pp. 181-192.

[25] A. Ferrante, G. Marro, and L. Ntogramatzidis, A parameterization of the solutions of the finite-horizon $L Q$ problem with general cost and boundary condition, Automatica, 41:8(2005), pp. 1359-1366.

[26] R. Cogburn and H.T. Davis, Periodic Splines and Spectral Estimation, The Annals of Statistics, 2:6(1974), pp. 1108-1126.

[27] H. Kano, M. Egerstedt, H. Fujioka, S. Takahashi, and C.F. Martin, Periodic Smoothing Splines, Automatica, no. 44, pp. 182-192, 2008.

[28] S. Takahashi And C.F. Martin, Optimal Control Theoretic Splines and Its Application to Mobile Robot, Proc. of the IEEE International Conference on Control Applications (CA), 2004.

[29] A.Z. Averbuch, A.B. Pevnyi, And V.A. Zheludev, Butterworth wavelet transforms derived from discrete interpolatory splines: recursive implementation, Signal Processing, 81(2001), pp. 2363-2382.

[30] W.M. Wonham, On a Matrix Riccati Equation of Stochastic Control, SIAM Journal of Control and Optimization, 6(1968), pp. 681-697.

[31] S. Bittanti, P. Colaneri, and G. Guardabassi, Analysis of the Periodic Lyapunov and Riccati Equations via Canonical Decomposition, SIAM Journal on Control and Optimization, 24(1986), pp. 1138-1149.

[32] I. Kiguradze, On Periodic-Type Solutions of Systems of Linear Ordinary Differential Equations, Abstract and Applied Analysis, 5(2004), pp. 395-406. 


\section{Appendix A.}

Proof of Proposition 3.1. From the dynamical constraints (2) it is obtained that

$$
x(t)=e^{A t} x(0)+\int_{0}^{t} e^{A(t-s)} B u(s) d s,
$$

which, inserting the matrices and vectors of (5), is

$$
\left(\begin{array}{c}
x_{1}(t) \\
x_{2}(t)
\end{array}\right)=\left(\begin{array}{c}
x_{1}(0)+t x_{2}(0)+\int_{0}^{t}(t-s) u(s) d s \\
x_{2}(0)+\int_{0}^{t} u(s) d s
\end{array}\right) .
$$

At the terminal time, we obtain

$$
\left(\begin{array}{c}
x_{1}(T) \\
x_{2}(T)
\end{array}\right)=\left(\begin{array}{c}
x_{1}(0)+T x_{2}(0)+\int_{0}^{T}(T-s) u(s) d s \\
x_{2}(0)+\int_{0}^{T} u(s) d s
\end{array}\right),
$$

and since $x(0)=x(T)$ this yields

$$
\begin{aligned}
x_{2}(0) & =-\frac{1}{T} \int_{0}^{T}(T-s) u(s) d s \\
\int_{0}^{T} u(s) d s & =0 .
\end{aligned}
$$

It follows from (68) that the only feasible constant control is $\bar{u}^{*} \equiv 0$. For $\bar{u}^{*} \equiv 0$, the spline is $\bar{x}_{1}^{*} \equiv \bar{z}=\frac{1}{N} \sum_{i=1}^{N} z_{i}$. Therefore $\bar{J}^{*}=J(0, \bar{z})$.

Denote by $\hat{x}_{1}(t)$ the periodic, cubic spline that interpolates the points $\left(z_{i}-\bar{z}\right)$. This spline exists and is unique for distinct sampling angles $t_{i}$, and if there is at least one $z_{i} \neq \bar{z}$ in the set, $\hat{x}_{1}(t) \not \equiv \bar{z}$.

It follows that $\hat{u}(t)=\ddot{\hat{x}}_{1}(t)$ is well defined, non-zero and lies in the feasible region of Problem 2.1. Now let $\alpha \in \mathbb{R}$ and consider

$$
\begin{aligned}
\Gamma(\alpha) & =J(\alpha \hat{u}(t), \alpha \hat{x}(t)+\bar{z}) \\
& =\frac{\alpha^{2}}{2} \int_{0}^{T} \hat{u}(t)^{2} d t+\frac{\varepsilon^{2}}{2} \sum_{i=1}^{N}\left(t_{i}-t_{i-1}\right)\left(z_{i}-\alpha \hat{x}_{1}\left(t_{i}\right)-\bar{z}\right)^{2} \\
& =\frac{\alpha^{2}}{2} \int_{0}^{T} \hat{u}(t)^{2} d t+\frac{\varepsilon^{2}}{2} \sum_{i=1}^{N}\left(t_{i}-t_{i-1}\right)(1-\alpha)^{2}\left(z_{i}-\bar{z}\right)^{2} .
\end{aligned}
$$

The derivative of $\Gamma(\alpha)$ at $\alpha=0$ is

$$
\begin{aligned}
\frac{\partial \Gamma(\alpha)}{\partial \alpha}=\alpha \int_{0}^{T} \hat{u}(t)^{2} d t-\varepsilon^{2}(1-\alpha) \sum_{i=1}^{N}\left(t_{i}-\right. & \left.t_{i-1}\right)\left(z_{i}-\bar{z}\right)^{2} \\
& =-\varepsilon^{2} \sum_{i=1}^{N}\left(t_{i}-t_{i-1}\right)\left(z_{i}-\bar{z}\right)^{2}<0 .
\end{aligned}
$$

It follows that there exists an $\alpha^{*}$ such that $\Gamma\left(\alpha^{*}\right)<\Gamma(0)$. 
Proof of Proposition 3.5. Note that if (60) - (61) hold, it follows that

$$
u_{T}^{N}(T)=u_{T}^{N}(0)
$$

Therefore, if we can show that (50), (52) and (54) correspond to (37), (38) and (39) in the limit $N \rightarrow \infty$, the proposition is proved.

\section{Convergence for $P$.}

For simplicity, assume the data is equidistant and introduce the notation $\Delta t=t_{i+1}-$ $t_{i}$. Further, according to the standard dynamic programming approach, the Riccati equation is solved by backwards integration starting at $t_{N}$. Therefore we can write $t_{N-i}=t_{N}-i \Delta t$. Introduce the notation

$$
\begin{aligned}
P^{N}(t) & \triangleq P_{i}(t) \quad t \in\left(t_{i}, t_{i+1}\right], \quad i=0, \ldots, N-1 \\
F(P) & \triangleq-A^{\prime} P-P A+P B Q B^{\prime} P .
\end{aligned}
$$

Consider the continuous function $P(t)$ defined by (37). Taylor expansion of $P(t)$ around $t=t_{i+1}$ yields

$$
P\left(t_{i}\right)=P\left(t_{i+1}\right)-\Delta t F\left(P\left(t_{i+1}\right)\right)+\Delta t C^{\prime} R^{-1} C+O\left(\Delta t^{2}\right) .
$$

Now consider the function $P^{N}$. An expression corresponding to (74) for $P^{N}$ is obtained by, for each interval $\left(t_{i}, t_{i+1}\right]$, integrating the differential equation (50) backwards and then adding the correction term:

$$
P^{N}\left(t_{i}\right)=P^{N}\left(t_{i+1}\right)-\Delta t F\left(P^{N}\left(t_{i+1}\right)\right)+\Delta t C^{\prime} R^{-1} C+O\left(\Delta t^{2}\right) .
$$

Introduce $\Delta P \triangleq P^{N}-P$. Then

$$
\Delta P\left(t_{i}\right)=\Delta P\left(t_{i+1}\right)+\Delta t\left(F\left(P^{N}\left(t_{i+1}\right)\right)-F\left(P\left(t_{i+1}\right)\right)\right)+O\left(\Delta t^{2}\right) .
$$

For constant $A, B$ and $Q$ the function $F(\cdot)$ is Lipschitz in a neighborhood of $P$, i.e. it holds for some constant $K$ that

$$
\left\|F\left(P^{N}\right)-F(P)\right\| \leq K\left\|P^{N}-P\right\| .
$$

Thus

$$
\left\|\Delta P\left(t_{i}\right)\right\| \leq(1+\Delta t K)\left\|\Delta P\left(t_{i+1}\right)\right\|+k \Delta t^{2} \leq e^{K \Delta t}\left\|\Delta P\left(t_{i+1}\right)\right\|+k \Delta t^{2}
$$

for some constants $K$ and $k$. Therefore, starting the integration at the same endpoint for $P$ and $P^{N}$ so that $\Delta P\left(t_{N}\right)=0$, we get

$$
\begin{aligned}
\left\|\Delta P\left(t_{N-1}\right)\right\| & \leq k \Delta t^{2} \\
\left\|\Delta P\left(t_{N-2}\right)\right\| & \leq\left(1+e^{K \Delta t}\right) k \Delta t^{2} \\
\vdots & \\
\left\|\Delta P\left(t_{N-i}\right)\right\| & \leq\left(1+e^{K \Delta t}+\left(e^{K \Delta t}\right)^{2}+\cdots+\left(e^{K \Delta t}\right)^{i-1}\right) k \Delta t^{2} \\
& =\frac{e^{i K \Delta t}-1}{e^{K \Delta t}-1} k \Delta t^{2} \leq \frac{e^{i K \Delta t}-1}{K \Delta t} k \Delta t^{2} \leq \frac{e^{K T}-1}{K} k \Delta t .
\end{aligned}
$$


Now fix the time $t^{*} \in[0, T]$ and let $t_{N-i}=t^{*}$. If more data points are added so that $N$ grows, $i$ will grow since $t^{*}$ is fix, while $\Delta t$ decreases. Therefore

$$
\lim _{i \rightarrow \infty}\left\|\Delta P\left(t^{*}\right)\right\|=0
$$

The result also holds for unequally spaced data, in which case the convergence rate is bounded by the largest interval $t_{i+1}-t_{i}$. Convergence for $\phi$ and $s$ can be shown by a similar argument. 
